Article

\title{
Quantum Magneto-Optics in Graphene
}

\author{
Leonid Falkovsky ${ }^{1,2}$ \\ ${ }^{1}$ Landau Institute for Theoretical Physics, Moscow reg., Chernogolovka, av. Semenov 1a, \\ 142432 Russia \\ ${ }^{2}$ Verechagin Institute of the High Pressure Physics, Troitsk 142190, Russia; E-Mail: falk@itp.ac.ru
}

Received: 11 December 2014 / Accepted: 25 December 2014 / Published: 5 January 2015

\begin{abstract}
The optical conductivity of graphene in quantizing magnetic fields is studied. Both dynamical conductivities, longitudinal and Hall's, are analytically evaluated. The conductivity peaks are explained in terms of electron transitions. The optical transitions obey the selection rule with $\Delta n=1$ for the Landau number $n$. The light transmission and Faraday rotation in the quantizing magnetic fields are calculated.
\end{abstract}

Keywords: transmission; Faraday rotation; graphene

\section{Introduction}

Comprehensive literature on the graphene family is expressed usually in terms of the Dirac gapless fermions. According to this picture, there are two bands at the $K$ hexagon vertexes of the Brillouin zone without any gap between them, and the electron dispersion can be considered as linear in the wide wave-vector region. For the dispersion linearity, this region should be small compared with the size of the graphene Brillouin zone, i.e., less than $10^{-8} \mathrm{~cm}^{-1}$, providing the small carrier concentration $n \ll 10^{16} \mathrm{~cm}^{-1}$. Pristine graphene at zero temperature has no carriers, and the Fermi level should divide the conduction and valence bands. However, undoped graphene cannot be really obtained, and so far, the purest graphene contains about $n \sim 10^{9} \mathrm{~cm}^{-2}$ of carriers. Then, the following problem appears: how do Coulomb electron-electron interactions renormalize the linear dispersion, and does graphene become an insulator with a gap?

Semiconductors with a gap are needed for electronic applications. Investigations of the graphene bilayer and multilayer are very popular, as the gap appears when the bias is applied. We see how physics made a circle for a half century, returning to graphite studies. Here, Slonczewski, Weiss and McClure 
(SWMC) should be mentioned, because they have stated the description of a layered matter [1] with interactions strong in the layer and weak between layers.

The most accurate investigation of the band structure of metals and semiconductors is a study of the Landau levels through experiments, such as magneto-optics [2-10], magneto-transport [11-15] and magnetic oscillation of the optical phonon [16,17]. In magnetic fields, the classical and quantum Hall effects are observed, as well as the polarization rotation for transmitted (Faraday rotation) or reflected light (Kerr rotation). However, the interpretation of the experimental results involves a significant degree of uncertainty, because it is not clear how the resonances can be identified and to which electron transitions they correspond.

The theoretical solution for the band problem in magnetic fields often cannot be exactly found. A typical example is presented by graphene layers. For bilayer graphene and graphite, the effective Hamiltonian is a $4 \times 4$ matrix, giving four energy bands. The trigonal warping described by the effective Hamiltonian with a relatively small parameter $\gamma_{3}$ provides an evident effect. Another important parameter is the gate-tunable bandgap $U$ in bilayer graphene. In this situation, the quantization problem cannot be solved within a rigorous method. To overcome this difficulty, several methods have been proposed for approximate [9,18-21], numerical [22-26] and semiclassical quantization [27,28].

The present paper is organized as follows. In Section 2, we recall the electron dispersion in graphene. In Section 3, we describe in detail the quantization in magnetic fields. The optical conductivity, light transmission, and Faraday rotation are discussed in Section 4.

\section{Electron Dispersion in Graphene}

The symmetry of the $K$ point is $C_{3 v}$ with the three-fold axis and reflection planes. This group has a two-fold representation with the basis functions transforming each in other under reflections and obtaining the factors $\exp ( \pm 2 \pi i / 3)$ in rotations. The linear momentum variations from the $K$ point $p_{ \pm}=\mp i p_{x}-p_{y}$ transform in a similar way. The effective Hamiltonian is invariant under the group transformations, and we have the unique possibility to construct the invariant Hamiltonian linear in the momentum as:

$$
H(\mathbf{p})=\left(\begin{array}{cc}
0 & v p_{+} \\
v p_{-} & 0
\end{array}\right)
$$

where $v$ is a constant of the velocity units. The same Hamiltonian was written using the tight binding model.

The eigenvalues of this matrix give two bands:

$$
\varepsilon_{1,2}=\mp v \sqrt{p_{x}^{2}+p_{y}^{2}}=\mp v p
$$

where the sign $\mp$ corresponds to holes and electrons. The gapless linear spectrum arises as a consequence of the symmetry, and the chemical potential at zero temperatures coincides with the band crossing due to the carbon valence. The cyclotron mass has the form:

$$
m(\varepsilon)=\frac{1}{2 \pi} \frac{d S(\varepsilon)}{d \varepsilon}=\frac{\varepsilon}{v^{2}}
$$

and the carrier concentration at zero temperature: $n(\mu)=\mu^{2} / \pi \hbar^{2} v^{2}$ is expressed in terms of the chemical potential $\mu$. 
Tuning the gate voltage, the linearity of the spectrum has been examined in the Shubnikov-de Haas studies [29] with the help of the connection between the effective mass and the carrier concentration at the Fermi level $m(\mu) v=\mp \hbar \sqrt{\pi n(\mu)}$. The "constant" parameter $v$ was found to be no longer constant, but at low carrier concentrations $n \sim 10^{9} \mathrm{~cm}^{-2}$, it exceeds its usual value $v=1.05 \pm 0.1 \times 10^{8} \mathrm{~cm} / \mathrm{s}$ (at concentrations $n>10^{11} \mathrm{~cm}^{-2}$ ) by a factor of three.

This is a result of electron-electron interactions, which becomes stronger at low carrier concentrations. The logarithmic renormalization of the velocity was found by Abrikosov and Beneslavsky in [30] for the three-dimensional case and in [31,32] for two-dimensional graphene. Notice that no phase transition was revealed, even at the lowest carrier concentration. We can conclude that the Coulomb interactions do not create any gap in the spectrum.

\section{Graphene in Magnetic Fields}

In the presence of the magnetic field $B$, the momentum projections $p_{+}$and $p_{-}$become the operators with the commutation rule $\left\{\hat{p}_{+}, \hat{p}_{-}\right\}=-2 e \hbar B / c$. We use the relations:

$$
v \hat{p}_{+}=\omega_{B} a, \quad v \hat{p}_{-}=\omega_{B} a^{+}
$$

involving the creation $a^{+}$and annihilation $a$ operators with $\omega_{B}=v \sqrt{2|e| \hbar B / c}$. We will write only one of two $x, y$ space coordinates, including the corresponding degeneracy proportional to the magnetic field in the final results.

For graphene, we search the eigenfunction of Hamiltonian (1) in the form:

$$
\psi_{s n}^{\alpha}(x)=\left\{\begin{array}{c}
C_{s n}^{1} \varphi_{n-1}(x) \\
C_{s n}^{2} \varphi_{n}(x)
\end{array}\right.
$$

where $\varphi_{n}(x)$ are orthonormal Hermitian functions with the Landau number $n \geq 0$. Canceling the Hermitian functions from the equations, we obtain a system of linear equations for the eigenvector $\mathbf{C}_{s n}$ :

$$
\left(\begin{array}{cc}
-\varepsilon & \omega_{B} \sqrt{n} \\
\omega_{B} \sqrt{n} & -\varepsilon
\end{array}\right) \times\left\{\begin{array}{l}
C_{s n}^{1} \\
C_{s n}^{2}=0
\end{array}\right.
$$

giving the eigenvalues:

$$
\varepsilon_{s n}=\mp \omega_{B} \sqrt{n}
$$

with $s=1,2$ and $n=0,1,2 \ldots$ For $n=0$, there is only one level $\varepsilon_{10}=0$ with $C_{0}^{1}=0, C_{0}^{2}=1$, as follows from Equation (2). The wave function columns are written:

$$
\begin{aligned}
& C_{s n}^{1} \\
& C_{s n}^{2}
\end{aligned}=\frac{1}{\sqrt{2}}\left\{\begin{array}{c}
1 \\
-1
\end{array} \text { and } \begin{array}{l}
1 \\
1
\end{array}\right.
$$

for $s=1$ and $s=2$ and $n=1,2 \ldots$ Levels and electron transitions are shown in Figure 1. 


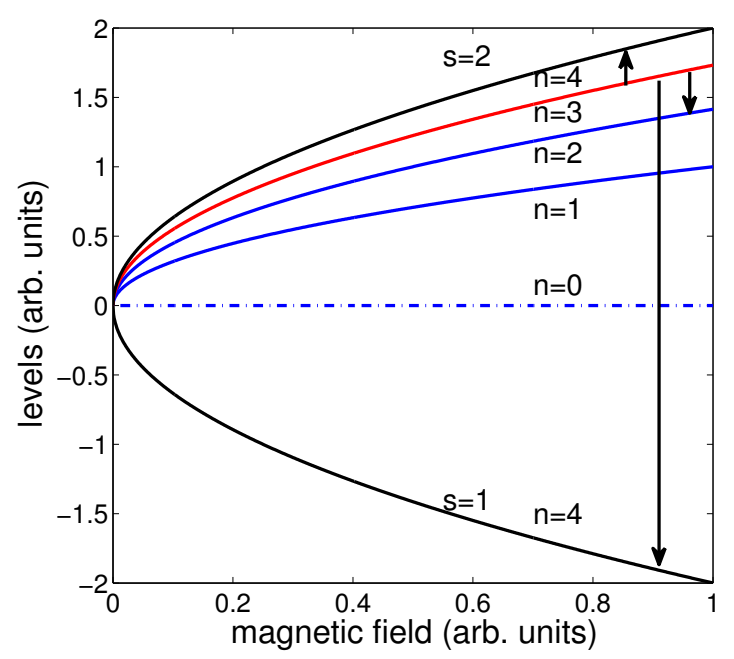

Figure 1. Magnetic levels in graphene and electron transitions involve the level with $n=3$ in the conduction band, $s=2$.

\section{Magneto-Optic Effects in Graphene}

An important peculiarity of conductivities in the presence of magnetic fields is an appearance of the Hall component $\sigma_{x y}(\omega)$. The Hall conductivity violates the rotation symmetry of graphene around the major axis. This implies the rotation of the linear polarized electromagnetic wave, i.e., the Faraday and Kerr effects for transmitted and reflected waves, correspondingly. Electron transitions are possible between the levels with the neighboring Landau numbers $n$ and various bands $s$, and therefore, the resonance denominators $\Delta_{s s^{\prime} n}=\varepsilon_{s n}-\varepsilon_{s^{\prime}, n+1}$ arise in the conductivity tensor.

Calculations [18] give the conductivities for graphite in the collisionless limit when the electron collision frequency $\Gamma$ is much less than the level splitting:

$$
\begin{aligned}
& \left.\begin{array}{c}
\sigma_{x x}(\omega) \\
i \sigma_{x y}(\omega)
\end{array}\right\}=i \sigma_{0} \frac{\omega_{B}^{2}}{\pi^{2}} \sum_{n, s, s^{\prime}} \frac{\Delta f_{s s^{\prime} n}}{\Delta_{s s^{\prime} n}} \\
& \times\left[\left(\omega+i \Gamma+\Delta_{s s^{\prime} n}\right)^{-1} \pm\left(\omega+i \Gamma-\Delta_{s s^{\prime} n}\right)^{-1}\right]
\end{aligned}
$$

Here, $\Delta f_{s s^{\prime} n}=f\left(\varepsilon_{s^{\prime} n+1}\right)-f\left(\varepsilon_{s n}\right)$ is the difference of the Fermi functions and:

$$
\sigma_{0}=e^{2} / 4 \hbar
$$

is the universal graphene conductivity.

For low carrier concentrations, e.g., for the low chemical potential, the electron transitions take place between the certain Landau levels obeying the condition $\Delta n=1$. For instance, the Landau levels at 7 Tin the upper band are $\varepsilon_{2, n}=0,98,138$, and $169 \mathrm{meV}$ for $n=0$ to $n=3$ and in the low band $\varepsilon_{1, n}=0,-98,-138$, and $-169 \mathrm{meV}$.

The transmission coefficient:

$$
T=1-\frac{4 \pi}{c} \operatorname{Re} \sigma_{x x}
$$

and the Faraday rotation angle:

$$
\Theta_{F}=\frac{2 \pi}{c} \operatorname{Re} \sigma_{x y}
$$


calculated for those cases when the chemical potential increases and the carriers fill sequentially the levels from $n=0$ to $n=3$ are shown in Figures 2 and 3. For instance, if the level with $n=0$ is occupied, the transition into the level $|2,1\rangle$ is possible (at $98 \mathrm{meV}$ in Figures), as well as from the level $|1,2\rangle$ (at $236 \mathrm{meV}$, is not shown in the Figures). We use the notation $|s, n\rangle$ for the levels with the band symbol $s=1,2$ and the Landau number $n$.

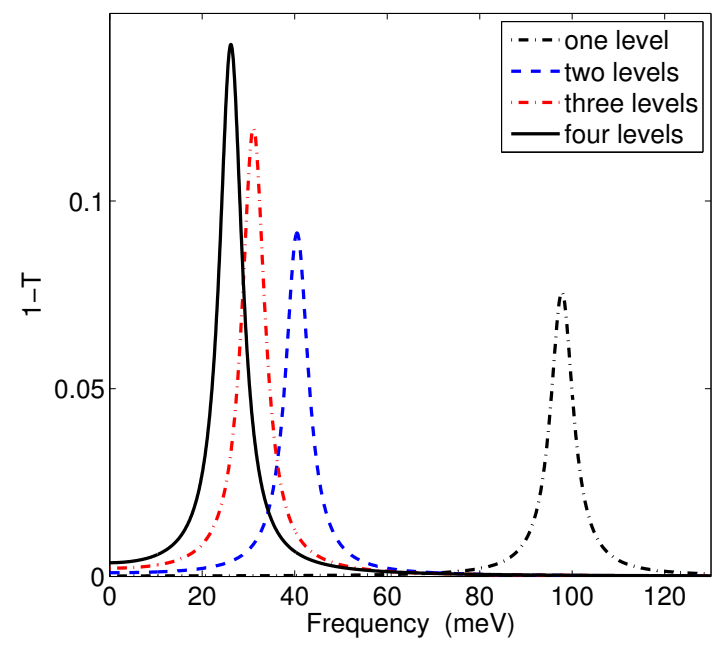

Figure 2. Light transmission through graphene with low carrier concentrations when carriers fill only one, two, three or four levels.

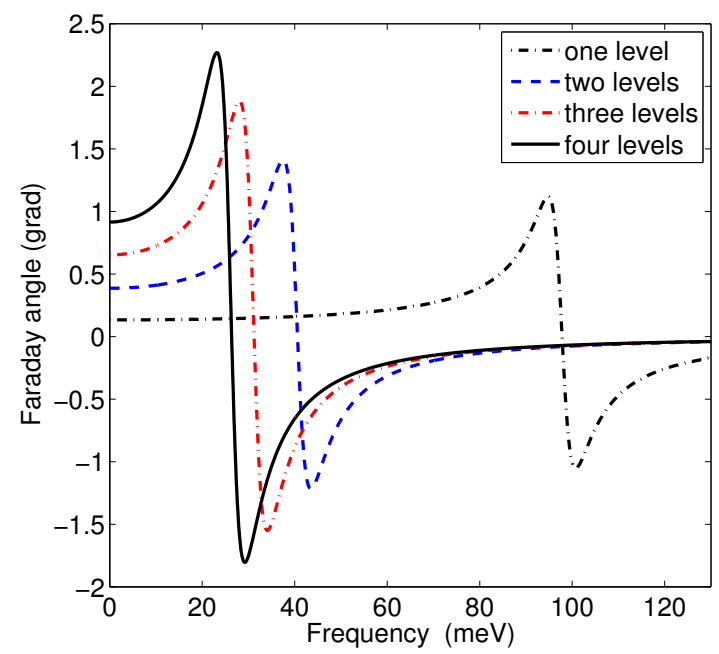

Figure 3. Faraday rotation for the cases when electrons occupy only several levels; the electron relaxation frequency $\Gamma=3 \mathrm{meV}$.

At high carrier concentrations, the chemical potential $\mu$ is much larger than the intraband level splitting $\Delta_{s s n}=e B / c m(\varepsilon) \equiv \Omega$, called the cyclotron frequency, with the effective mass $m(\varepsilon)=\varepsilon / v^{2}$, and the conductivities (3) coincide with the semiclassical Drude conductivities:

$$
\left.\begin{array}{l}
\sigma_{x x}(\omega) \\
\sigma_{x y}(\omega)
\end{array}\right\}=\sigma_{0} \frac{4 v^{2}}{\pi \hbar} \int d \varepsilon\left(-\frac{d f_{0}}{d \varepsilon}\right) \frac{m(\varepsilon)}{\omega^{* 2}-\Omega^{2}} \times\left\{\begin{array}{c}
i \omega^{*} \\
\Omega
\end{array}\right.
$$

with $\omega^{*}=\omega+i \Gamma$. 
At low temperatures $T<<\Gamma$, we can integrate using the $\delta$-function, $d f_{0} / d \varepsilon=-\delta(\varepsilon-\mu)$,

$$
\left.\begin{array}{l}
\sigma_{x x}(\omega) \\
\sigma_{x y}(\omega)
\end{array}\right\}=\frac{4 \sigma_{0} \mu}{\pi \hbar\left(\omega^{* 2}-\Omega^{2}\right)} \times\left\{\begin{array}{c}
i \omega^{*} \\
\Omega
\end{array}\right.
$$

where the cyclotron frequency $\Omega=e B v^{2} / c \mu$ is taken on the Fermi surface. The high carrier concentration case is illustrated in Figure 4.

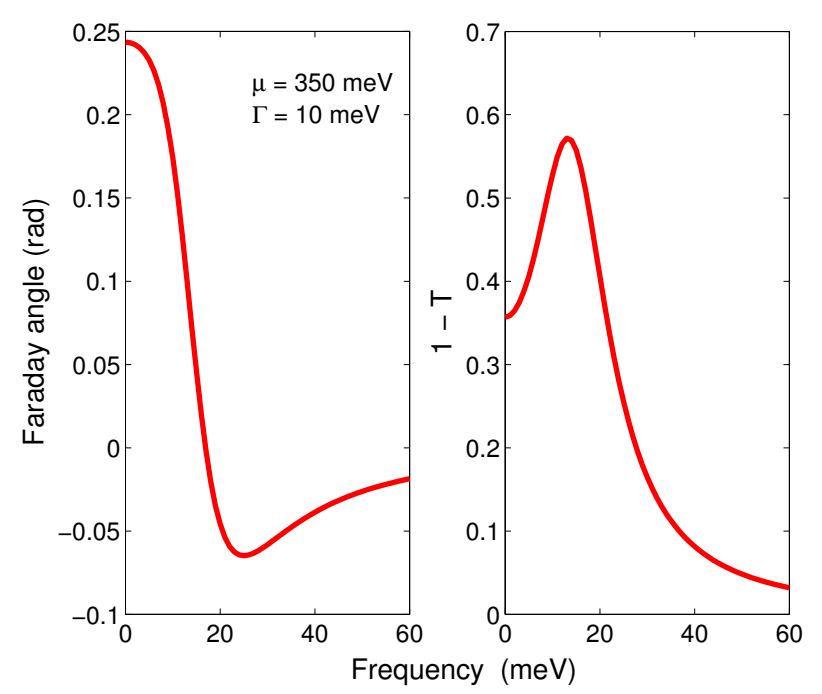

Figure 4. Faraday rotation in free suspended graphene with high carrier concentration at a magnetic field $7 \mathrm{~T}$; 13 levels in the upper electron band are below the Fermi level; the cyclotron frequency at the Fermi level $\Omega=13 \mathrm{meV}$; the electron relaxation frequency $\Gamma=10 \mathrm{meV}$.

\section{Discussion}

Graphene layers affect the transmission and the Faraday rotation in a linear order in the fine structure constant $\alpha$, whereas the reflected light intensity is quadratic to $\alpha$. Thus, we discuss here the characteristics of the transmitted light. Expressed linearly in conductivity $\sigma_{0}=e^{2} / 4 \hbar$, the transmission coefficient $T$ and the Faraday angle for free standing graphene are shown in Figures 2 and 3. The peaks in absorption (Figure 2) correspond to the electron transitions. The series of lines are observed in the $0.1-0.4 \mathrm{eV}$ interval. They are doublets excited by the electron transitions of the type $|1, n\rangle \rightarrow|2, n+1\rangle$ and $|1, n+1\rangle \rightarrow|2, n\rangle$ for $n>0$. All of these lines obey the selection rule $\Delta n=1$. Let us note that the transition frequencies in Figure 2 determine the maximum values of the derivative in the Faraday rotation (Figure 3).

\section{Conflicts of Interest}

The author declare no conflict of interest. 


\section{References}

1. Slonchewski, J.C.; Weiss, P.R. Band Structure of Graphite. Phys. Rev. 1958, 109, 272.

2. Suematsu, H.; Tanuma, S.I. Cyclotron Resonances in Graphite by Using Circularly Polarized Radiation. J. Phys. Soc. Jpn. 1972, 33, 1619.

3. Toy, W.W.; Dresselhaus, M.S.; Dresselhaus, G. Minority carriers in graphite and the H-point magnetoreflection spectra. Phys. Rev. B 1977, 15, 4077.

4. Doezema, R.E.; Datars, W.R.; Schaber, H.; Van Schyndel, A. Far-infrared magnetospectroscopy of the Landau-level structure in graphite. Phys. Rev. B 1979, 19, 4224.

5. Mendez, E.; Misu, A.; Dresselhaus, M.S. Magnetoreflection study of graphite under pressure. Phys. Rev. B 1980, 21, 827.

6. Li, Z.Q.; Tsai, S.W.; Padilla, W.J.; Dordevic, S.V.; Burch, K.S.; Wang, Y.J.; Basov, D.N. Infrared probe of the anomalous magnetotransport of highly oriented pyrolytic graphite in the extreme quantum limit.. Phys. Rev. B 2006, 74, 195404.

7. Orlita, M.; Faugeras, C.; Martinez, G.; Maude, D.K.; Sadowski, M.L.; Potemski, M. Dirac Fermions at the H Point of Graphite: Magnetotransmission Studies. Phys. Rev. Lett. 2008, 100, 136403.

8. Orlita, M.; Faugeras, C.; Schneider, J.M.; Martinez, G.; Maude, D.K.; Potemski, M. Graphite from the Viewpoint of Landau Level Spectroscopy: An Effective Graphene Bilayer and Monolayer. Phys. Rev. Lett. 2009, 102, 166401.

9. Orlita, M.; Potemski, M. Dirac electronic states in graphene systems: optical spectroscopy studies. Semicond. Sci. Technol. 2010, 25, 063001.

10. Crassee, I.; Levallois, J.; Walter, A. L.; Ostler, M.; Bostwick, A.; Rotenberg, E.; Seyler, T.; van der Marel, D.; Kuzmenko, A. Giant Faraday rotation in single- and multilayer graphene. Nat. Phys. 2011, 7, 48.

11. Kopelevich, Y.; Torres, J.H.S.; da Silva, R.R.; Mrowka, F.; Kempa, H.; Esquinazi, P. Reentrant Metallic Behavior of Graphite in the Quantum Limit. Phys. Rev. Lett. 2003, 90, 156402.

12. Luk'yanchuk, I.A.; Kopelevich, Y. Dirac and Normal Fermions in Graphite and Graphene: Implications of the Quantum Hall Effect. Phys. Rev. Lett. 2006, 97, 256801.

13. Jiang, Z.; Zhang, Y.; Stormer, H.L.; Kim, P. Quantum Hall States near the Charge-Neutral Dirac Point in Graphene. Phys. Rev. Lett. 2007, 99, 106802.

14. Schneider, J.M.; Orlita, M.; Potemski, M.; Maude, D.K. Consistent Interpretation of the Low-Temperature Magnetotransport in Graphite Using the Slonczewski-Weiss-McClure 3D Band-Structure Calculations. Phys. Rev. Lett. 2009, 102, 166403.

15. Ramanayaka, A.N.; Mani, R.G. Transport study of the Berry phase, resistivity rule, and quantum Hall effect in graphite. Phys. Rev. B 2010, 82, 165327.

16. Ando, T. Magnetic Oscillation of Optical Phonon in Graphene. J. Phys. Soc. Jpn. 2007, 76, 024712 .

17. Goerbig, M.O.; Fuchs, J.N.; Kechedzhi, K.; Fal'ko, V.I. Filling-Factor-Dependent Magnetophonon Resonance in Graphene. Phys. Rev. Lett. 2007, 99, 087402.

18. Falkovsky, L.A. Quantum magneto-optics of graphite with trigonal warping. Phys. Rev. B 2011,84, 115414. 
19. Li, G.; Andrei, E.Y. Observation of Landau levels of Dirac fermions in graphite. Nat. Phys. 2007, $3,623$.

20. Chuang, K.C.; Baker, A.M.R.; Nicholas, R.J. Magnetoabsorption study of Landau levels in graphite. Phys. Rev. B 2009, 80, 161410(R).

21. Zhang, L.M.; Li, Z.Q.; Basov, D.N.; Fogler, M.M.; Hao, Z.; Martin, M.C. Determination of the electronic structure of bilayer graphene from infrared spectroscopy. Phys. Rev. B 2008, 78, 235408.

22. Ushio, H.; Uda, T.; Uemura, Y. Theory of Cyclotron Resonance of Graphite. I. Determination of the Degree of Band Warping. J. Phys. Soc. Jpn. 1972, 33, 1551.

23. Nakao, K. Landau Level Structure and Magnetic Breakthrough in Graphite. J. Phys. Soc. Jpn 1976, 40,761.

24. Partoens, B.; Peeters, F.M. From graphene to graphite: Electronic structure around the K point. Phys. Rev. B 2006, 74, 075404.

25. Grüneis, A.; Attaccalite, C.; Wirtz, L.; Shiozawa, H.; Saito, R.; Pichler, T.; Rubio, A. Tight-binding description of the quasiparticle dispersion of graphite and few-layer graphene. Phys. Rev. B 2008, $78,205425$.

26. Kuzmenko, A.B.; Crassee, I.; van der Marel, D.; Blake, P.; Novoselov, K.S. Determination of the gate-tunable band gap and tight-binding parameters in bilayer graphene using infrared spectroscopy. Phys. Rev. B 2009, 80, 165406.

27. Falkovsky, L.A.; Eksp, Z. Quasiclassical Quantization of Electrons and Holes in Bismuth in a Magnetic Field. Teor. Fiz. 1965, 49, 609.

28. Dresselhaus, G. Graphite Landau levels in the presence of trigonal warping. Phys. Rev. B 1974, 10, 3602.

29. Elias, D.C.; Gorbachev, R.V.; Mayorov, A.S.; Morozov, S.V.; Zhukov, A.A.; Blake, P.; Ponomarenko, L.A.; Grigorieva, I.V.; Novoselov, K.S.; Guinea, F.; et al. Dirac cones reshaped by interaction effects in suspended graphene. Nat. Phys. 2011, 7, 701.

30. Abrikosov, A.A.; Beneslavsky, S.D. Possible Existence of Substances Intermediate Between Metals and Dielectrics. Sov. Phys. JETP 1971, 32, 699.

31. Gonzalez, J.; Guinea, F.; Vozmediano, M.A.H. Non-Fermi liquid behaviour of electrons in the half-filled honeycomb lattice (A renormalization group approach). Nucl. Phys. B 1994, 424, 595.

32. Mishchenko, E.G. Effect of Electron-Electron Interactions on the Conductivity of Clean Graphene. Phys. Rev. Lett. 2007, 98, 216801.

(C) 2015 by the author; licensee MDPI, Basel, Switzerland. This article is an open access article distributed under the terms and conditions of the Creative Commons Attribution license (http://creativecommons.org/licenses/by/4.0/). 